\title{
Interacting electrons in one dimension beyond the Luttinger-liquid limit
}

\author{
Gilad Barak ${ }^{1}$, Hadar Steinberg ${ }^{2}$, Loren N. Pfeiffer ${ }^{3}$, Ken W. West ${ }^{3}$, Leonid Glazman ${ }^{4}$, Felix von Oppen ${ }^{5}$ \\ and Amir Yacoby ${ }^{1 \star}$
}

\begin{abstract}
Over the past several decades, Luttinger-liquid theory has provided a framework for interacting electrons in one dimension. However, the validity of the theory is strictly limited to low-energy excitations where the electron dispersion is linear. Interacting electrons in one-dimension beyond the Luttingerliquid limit, where the underlying dispersion of electrons is no longer linear, exhibit intriguing manifestations of the interactions, which have direct implications on many experimental systems. For example, consider the energy relaxation of particles or holes, the unoccupied states in a Fermi sea. Whereas in Luttinger-liquid theory such energy relaxation is strictly forbidden, in a nonlinearly dispersing one-dimensional electron system energy relaxation is allowed but very different for particles and holes. Here, we use momentum-resolved tunnelling to selectively inject energetic particles and holes into a quantum wire and study their relaxation processes. Our measurements confirm that energetic particles undergo fast relaxation to a thermalized distribution and holes retain their original injection energy, thereby providing a clear demonstration of electron dynamics beyond the Luttinger limit. A model of thermalization derived in the limit of weak interactions shows quantitative agreement with the experimental findings.
\end{abstract}

One-dimensional (1D) systems provide a unique venue for the study of interaction effects in many-body problems. The reduced dimensionality qualitatively changes the role of interactions, leading to such phenomena as spin-charge separation ${ }^{1}$, charge fractionalization $^{2}$ and Wigner crystallization ${ }^{3}$ (for a review, see ref. 4). Luttinger-liquid theory ${ }^{5}$ has been extremely successful in explaining and predicting most of these phenomena. However, the inherent underlying assumption of a linear dispersion limits the applicability of this theory to low-energy excitations. One physical process that is expected to show a qualitatively different behaviour when incorporating a nonlinear dispersion is energy relaxation. In three dimensions, the relaxation rate resulting from electronelectron interactions of excited particles or holes with energy $\varepsilon$ is proportional to $\left(\varepsilon-\varepsilon_{\mathrm{F}}\right)^{2} / \varepsilon_{\mathrm{F}}$, where $\varepsilon_{\mathrm{F}}$ is the Fermi energy, predicting a long lifetime for low-energy excitations (here and later, we use $\hbar=1$; ref. 6 ). Although this continuity across the Fermi surface was conjectured to be broken for some non-Fermi liquids ${ }^{7}$, the Luttinger-liquid theory manifestly preserves the particle-hole symmetry. However, in a 1D system where band parabolicity is not neglected, a fundamental difference arises between particles and holes $^{8,9}$. When a particle with initial momentum $k_{\mathrm{p}}\left(\left|k_{\mathrm{p}}\right|>\left|k_{\mathrm{F}}\right|\right)$ loses momentum $k$, it gives up an energy equal to $E_{\mathrm{p}}=\left(2 k_{\mathrm{p}} k-k^{2}\right) / 2 m^{*}$. Although $E_{\mathrm{p}}$ is too large to be carried by a single particle-hole $(\mathrm{p}-\mathrm{h})$ pair, relaxation is allowed through the excitation of multiple $\mathrm{p}-\mathrm{h}$ pairs (Fig. 1a). In marked contrast, the energy lost by an excited hole with initial momentum $k_{\mathrm{h}}\left(\left|k_{\mathrm{h}}\right|<\left|k_{\mathrm{F}}\right|\right)$ is $E_{\mathrm{h}}=\left(2 k_{\mathrm{h}} k+k^{2}\right) / 2 m^{*}$, too low to be carried by any number of p-h pairs (Fig. 1a). Here, we provide direct measurements of the relaxation properties of energetic particles and holes far from the Fermi energy, a regime where the conventional Luttinger-liquid theory does not apply.

A double-wire system (see Fig. 1b) is used to selectively inject electrons with a definite energy and momentum ${ }^{10}$ from the upper wire into the lower wire. Details on sample structure and measurement technique are provided in the Methods section. Owing to conservation of energy and momentum in the tunnelling process, tunnelling is allowed only when occupied states from the upper-wire dispersion overlap, in momentum and energy, with unoccupied states from the lower wire, or vice versa. Selective injection of electrons with specific energy and momentum is accomplished by inducing a relative displacement between the two dispersions in both momentum and energy. A bias, $V$, applied between the wires results in a displacement of $-e V$ in energy between the dispersions. A magnetic field, $B$, applied perpendicular to the plane formed by the two wires boosts the tunnelling electrons, and shifts the dispersion of the lower wire relative to the dispersion of the upper wire in momentum by an amount given by $-e B d$, where $d$ is the distance between the wires' centres. At sufficiently strong magnetic fields, $B^{\mathrm{L}(\mathrm{R})}=(-) 1 / e d\left|k_{\mathrm{F}}^{\mathrm{UW}}+k_{\mathrm{F}}^{\mathrm{LW}}\right|$, a right (left)moving electron from the upper wire (UW) tunnels into a left (right)-moving state in the lower wire (LW). The current resulting from this injection of directional electrons is then collected by the two drains, on the left and right of the source junction.

Figure $2 \mathrm{a}$ shows the total differential conductance for magnetic fields near $B^{\mathrm{L}}$ and $-e V$ ranging up to approximately $\pm E_{\mathrm{F}} / 3$. The strong cross-shaped differential-conductance peak corresponds to the onset of tunnelling when the Fermi point of one wire intersects the other wire's dispersion. Each of the four arms in this cross represents a distinct process. Arm (I) appears at $B>B^{\mathrm{L}}$ and $-e V>0$, so that the upper-wire Fermi point overlaps empty states in the lower wire. Along this branch, energetic particles are injected into the lower wire. Arm (IV), similarly, corresponds to the extraction of particles from the lower wire below the Fermi point, that is, the injection of energetic holes. We study dynamics in the lower wire, and therefore term the branch containing arms (I) and (IV) the 'hot' branch. The signal on the opposite diagonal (arms II and III) results from injection of particles and holes from the upper wire into the lower-wire Fermi point and will therefore be termed the cold branch. The total differential conductance along

${ }^{1}$ Department of Physics, Harvard University, Cambridge, Massachusetts, 02138, USA, ${ }^{2}$ Department of Condensed Matter Physics, Weizmann Institute of Science, Rehovot 76100, Israel, ${ }^{3}$ Bell Labs, Lucent Technologies, 700 Mountain Avenue, Murray Hill, New Jersey 07974, USA, ${ }^{4}$ Department of Physics, Yale University, New Haven, Connecticut 06520, USA, ${ }^{5}$ Dahlem Center for Complex Quantum Systems and Fachbereich Physik, Freie Universität Berlin, 14195 Berlin, Germany. *e-mail: yacoby@physics.harvard.edu. 
a

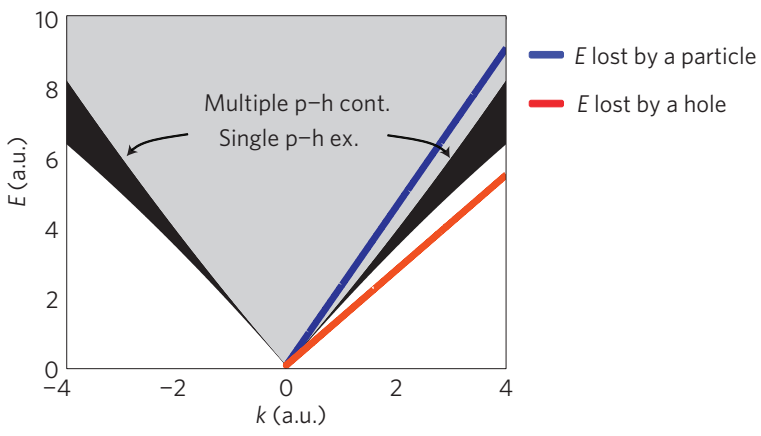

b

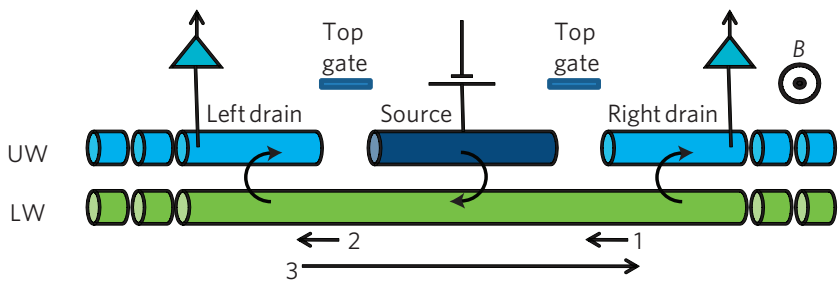

Figure 1 | Kinematic constraints on particle and hole relaxation in one dimension, and measurement set-up. $\mathbf{a}$, The energy lost by an energetic particle with momentum $k_{\mathrm{p}}, \Delta E=\left(2 k_{\mathrm{p}} k-k^{2}\right) / 2 m$, overlaps the continuum of multiple $\mathrm{p}$-h excitations, $\left(2 k_{\mathrm{F}}-k^{2}\right) / 2 m \leqslant E$, allowing for particle relaxation. As no such overlap exists for energetic holes with initial momentum $k_{\mathrm{h}}$, satisfying $\Delta E=\left(2 k_{\mathrm{h}} k+k^{2}\right) / 2 m$, these cannot relax. $\mathbf{b}$, The measurement set-up comprising two parallel quantum wires. Two top gates are used to locally deplete the upper wire (UW) directly below them, and define a short source junction and two long drains on both sides. Transport takes place by tunnelling from the upper wire to the lower wire (LW) at the source junction, and a second tunnelling event from the lower wire to the upper wire at the drains. The numbered arrows specify the position and direction of charge modes used in the theoretical description.

both the hot and cold branches is nearly independent of the type of process taking place.

Figure 2b,c shows the differential conductance measured separately at the right and left drains. At $B=B^{\mathrm{L}}$, a stronger signal is observed on the left drain (Fig. 2b) relative to the right drain (Fig. 2c). Information on the directionality of the injected current can be gained by subtracting the signals arriving to the right and left drains (Fig. 2d), showing a clear difference between energetic particles and holes. For most of the hot particle arm, the injected left-moving particles are indeed predominantly collected at the left drain. For the hot holes arm, as well as for the entire cold branch, a much smaller asymmetry is obtained.

The asymmetry between energetic particles and holes seen in the raw data presented in Fig. $2 \mathrm{~d}$ constitutes the main finding of this work. In the rest of this letter, we present a quantitative discussion of the results shown in Fig. 2, using a dimensionless measure of the asymmetry defined as

$$
A S=\frac{\mathrm{d} G^{\mathrm{L}}-\mathrm{d} G^{\mathrm{R}}}{\mathrm{d} G^{\mathrm{L}}+\mathrm{d} G^{\mathrm{R}}}
$$

with $\mathrm{d} G^{\mathrm{L}(\mathrm{R})}$ being the differential conductance measured at the left (right) drain. This is a normalized value that eliminates the effect of the source junction tunnelling efficiency. AS may range from -1 to 1 , with $|A S|=1$ when all of the current reaches only one drain, and $A S=0$ for an equal partitioning of the current between the two drains. In Fig. 3 a, we plot $A S$ versus $B$ along two trajectories marked by dashed lines in Fig. 2d, corresponding to the onset of injection of hot and cold particles to the lower wire. The difficulty in separating the cold and hot branches near their intersection point renders values of $A S$ in the range $-0.3 \mathrm{mV}<V_{\mathrm{SD}}<0.3 \mathrm{mV}$ difficult to interpret. We therefore focus our discussion on $\left|V_{\mathrm{SD}}\right|>0.3 \mathrm{mV}$.
We first consider the asymmetry profile of the cold branch. As described earlier, all points on this branch correspond to injection of electrons at the Fermi point of the lower wire. Nonetheless, the asymmetry (blue dots in Fig. 3a) clearly diminishes away from the crossing point, $B=B^{\mathrm{L}}$. This can be accounted for by considering the role of the drains in the measurement of current asymmetry. The upper wire is tuned to inject electrons into the Fermi point of the lower wire by application of a bias $V$. However, at the drains, where such bias is absent (because most of the bias drops on the source junction), both dispersions have the same Fermi energy, but except for $B=B^{\mathrm{L}(\mathrm{R})}$ there is no overlap between full and empty states of the two wires' dispersions (see insets (I), (II) of Fig. 3a). The imperfect tuning between upper-wire and lower-wire Fermi points at the drains along the cold branch reduces the tunnelling rate out of the lower wire and causes the directionality to be lost because of the inevitable presence of backscattering processes. We call this effect drain detuning, and define the degree of drain detuning as the momentum an electron has to acquire (or lose) to be collected by the drain, while conserving energy. Maximal asymmetry is therefore expected when the drains are exactly tuned to extract the charge from the lower wire. Indeed, from Fig. 3a we infer a maximal asymmetry for $B=B^{\mathrm{L}}=4.77 \mathrm{~T}$.

At low bias, the charge currents in the lower wire can be further analysed within the framework of Luttinger-liquid theory, which considers interacting electrons in the approximation of a linear dispersion. This assumed linearity yields a common velocity for all charge excitations, regardless of their energy, and allows for a wide applicability of the concept of charge fractionalization ${ }^{11-18}$. Measurements ${ }^{2}$ of the asymmetry of the injected current at low bias are consistent with that concept. Within the Luttinger-liquid paradigm, one may think of the charge of an electron injected with momentum close to one of the Fermi points as being fractionalized into counter-propagating excitations carrying charges $f=(1+g) / 2$ in the direction of the injected electron, and $1-f=(1-g) / 2$ in the opposite direction. Here $g$ is the Luttinger-liquid interaction parameter. Remarkably, the ratio

$$
A S /\left(G^{2 \mathrm{~T}} / G_{0}\right)=(2 f-1) / g=1
$$

is independent of the fractionalization (here $G^{2 \mathrm{~T}}$ is the twoterminal conductance measured between the two drains and $G_{0}=2 e^{2} / h$ is the conductance quantum). This conclusion, obtained within the Luttinger-liquid theory, is unambiguously confirmed by experiment ${ }^{2}$. In a linear d.c. measurement, the independence of the ratio equation (2) on $g$ is protected by momentum conservation ${ }^{18}$. In Fig. 3a we show an excellent fit between the asymmetry of cold particles and $G^{2 \mathrm{~T}}$, consistent with the previous findings ${ }^{2}$ (using methods described in ${ }^{10}$, we estimate $g=0.55$ giving $f=0.77$ ).

We now turn to study the richer picture obtained for energetic particles. We do not know a priori whether a particle or hole injected at an elevated energy maintains its energy, or rather relaxes part or all of it. In the absence of relaxation, a single peak in asymmetry is expected at $B=B^{\mathrm{L}}$, where particles are injected at the Fermi point of the source, and the drains are exactly tuned to extract them. In contrast, relaxed particles could give rise to an increased asymmetry through two mechanisms. First, particles with initial energy $\varepsilon_{\mathrm{I}}$ that relax part of their energy and reach $\varepsilon_{\mathrm{F}}<\varepsilon<\varepsilon_{\mathrm{I}}$ would show increased asymmetry, when the drain is better tuned to extract the relaxed particle. Second, the $\mathrm{p}-\mathrm{h}$ pairs that are excited in the relaxation process can also alter the measured asymmetry, because owing to the detuning, the drain preferentially extracts particles or holes. The latter process is described in detail below.

For hot particles $\left(B>B^{\mathrm{L}}\right)$ we indeed find the maximal asymmetry at a magnetic field larger than $B^{\mathrm{L}}$. As corroborated in the following, this is consistent with particle relaxation through creation of energetic $\mathrm{p}-\mathrm{h}$ pairs moving in the same direction as the relaxing 
a

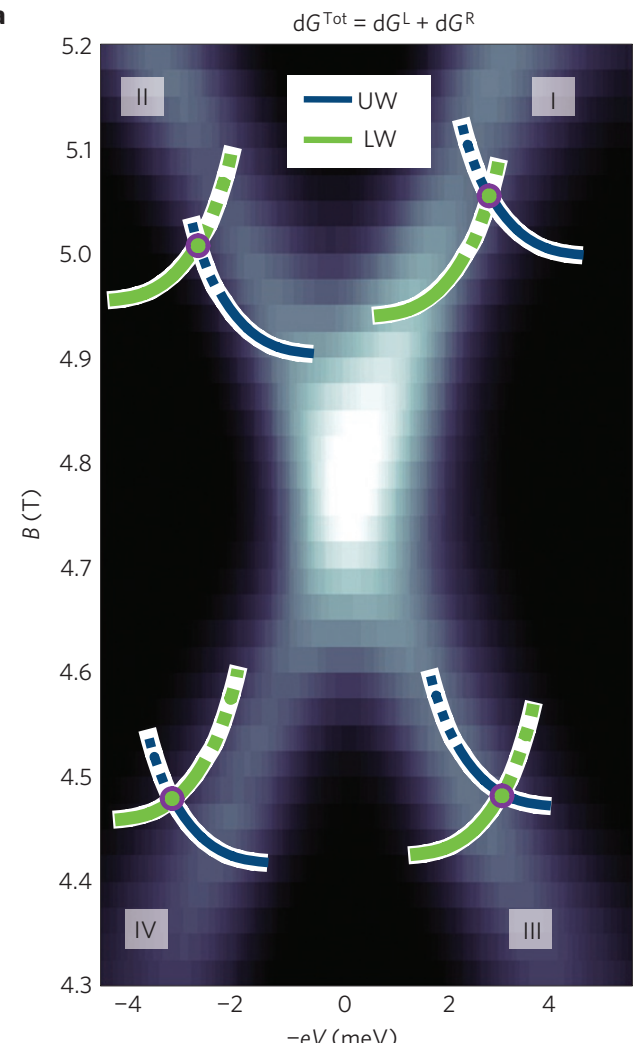

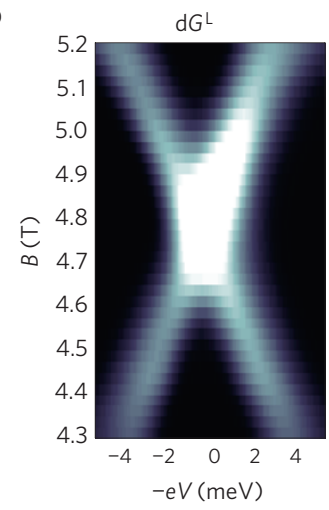

c

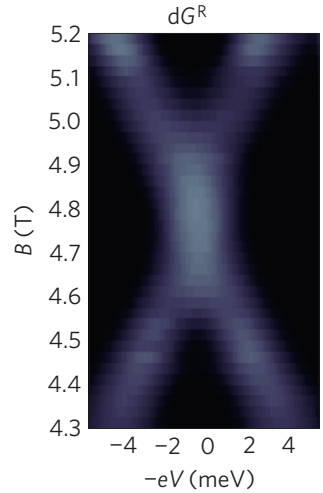

d

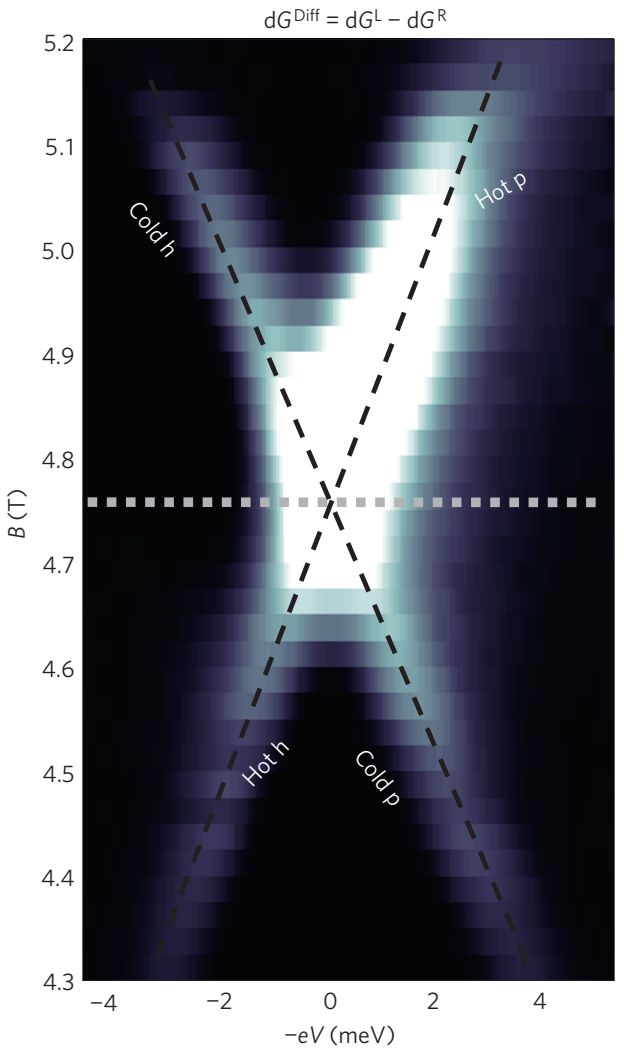

Figure 2 | Measured sum and difference of differential conductance on the two sides of the injection region. a, Total differential conductance. Each of the four branches of the conductance cross represents a different tunnelling process (see text). b,c, Differential conductance measured on the left drain, in the direction of the injected current (b), and on the right drain (c). $\mathbf{d}$, The difference between the differential conductance in the injection direction and counter to it reveals a strong $\mathrm{p}-\mathrm{h}$ asymmetry. The dashed lines mark the cold and hot branches. Along the grey dotted line $\left(B=B^{\mathrm{L}}\right)$, the drain is tuned to extract electrons from the Fermi point of the lower wire.

particle ${ }^{9}$. For energetic holes $\left(B<B^{\mathrm{L}}\right)$, we find an asymmetry equal to that of the cold branch. This suggests that the hot holes do not relax, so the drain detuning (Fig. $3 \mathrm{a}(\mathrm{IV})$ ) is $\Delta k$-the momentum offset at the drains between the lower-wire and upper-wire states at the injection energy. Importantly, to establish this conclusion for hot holes, we have to contrast it with an alternative, where the holes relax to $\varepsilon_{\mathrm{F}}$ by a mechanism that does not involve $\mathrm{p}-\mathrm{h}$ pair creation. As the drain dispersion intersects the lower-wire dispersion at the midpoint between the injection point and the Fermi point (open circle in Fig. $3 \mathrm{a}(\mathrm{IV})$ ), the absolute values of drain detuning $|\Delta k|$ are the same for complete energy relaxation to the Fermi level, and for no relaxation at all. To distinguish between complete and no relaxation, we study the asymmetry along a different $V-B$ trajectory in Fig. 2d, where the injection energy $(-e V)$ is changed while keeping $B$ constant at $B^{\mathrm{L}}$. Along this trajectory the drain is fixed to extract particles from the Fermi point of the lower wire independent of the injection energy (Fig. $3 \mathrm{~b}(\mathrm{I})$ ). The results, shown in Fig. 3b, present again a marked difference for the injection of energetic particles $(-e V>0)$ and holes $(-e V<0)$. For energetic particles we find that the asymmetry decreases initially but then remains constant independent of the injection energy. This is an indication that the injected particles relaxed close to the Fermi level. For energetic holes on the other hand a strong decrease in asymmetry is obtained, eliminating the possibility of full energy relaxation. As the possibility of partial energy relaxation for holes was ruled out, this observation decisively establishes that energetic holes did not relax at all during their transport in the lower wire before being collected at the drains. In the Supplementary Information we present yet another approach to establishing the relaxation rates of particles and holes that further corroborates this conclusion. The marked difference in relaxation times for particles and holes can be quantified, using the estimated time of transport through the lower wire. Particles travel a minimal distance of $\approx 2 \mu \mathrm{m}$ in the wire, as determined by the gates separating the source and drain junctions. For a typical Fermi velocity of $2 \times 10^{5} \mathrm{~m} \mathrm{~s}^{-1}$, it takes an electron $\sim 10^{-11} \mathrm{~s}$ to pass through the wire. Over that time particles do relax, whereas holes do not, which sets the limits for the relaxation times, $\tau_{\mathrm{e}}<10^{-11} \mathrm{~s}, \tau_{\mathrm{h}} \gg 10^{-11} \mathrm{~s}$, in the conditions of the experiment.

Although in the linear regime equation (2) remains true for any interaction strength, including the limit of weak interaction $(g \rightarrow 1, f \rightarrow 1)$, it may break down at higher bias, once the simplification of a linear spectrum assumed in Luttinger-liquid theory becomes invalid ${ }^{19}$. Indeed, our experiment shows a clear deviation from the predictions of Luttinger-liquid theory at higher bias. Although equation (2) holds when energetic holes are injected, the measured $A S$ for hot particles is significantly larger than $G^{2 \mathrm{~T}}$ (Fig. 3a). Below we show that this observation is quantitatively consistent with the notion of energy relaxation of hot particles, a process that does not exist under the assumptions of Luttinger-liquid theory.

To evaluate the asymmetry ratio equation (1) for the conditions of hot-particle injection (Fig. 3a(I)), we account for the nonlinear dispersion relation of electrons, in the weak interaction limit, $g \rightarrow 1$. In this limit, we can relate the currents of charge and energy in the lower wire, $I_{i}$ and $P_{i}$, respectively, to the electron distribution function,

$$
\begin{array}{r}
I_{i}=\left(G_{0} / e\right) \int\left[f_{i}(\varepsilon)-f_{0}(\varepsilon)\right] \mathrm{d} \varepsilon \\
P_{i}=\left(G_{0} / e^{2}\right) \int\left[f_{i}(\varepsilon)-f_{0}(\varepsilon)\right] \varepsilon \mathrm{d} \varepsilon
\end{array}
$$




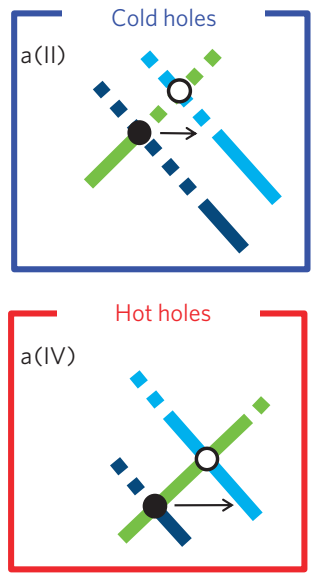

a
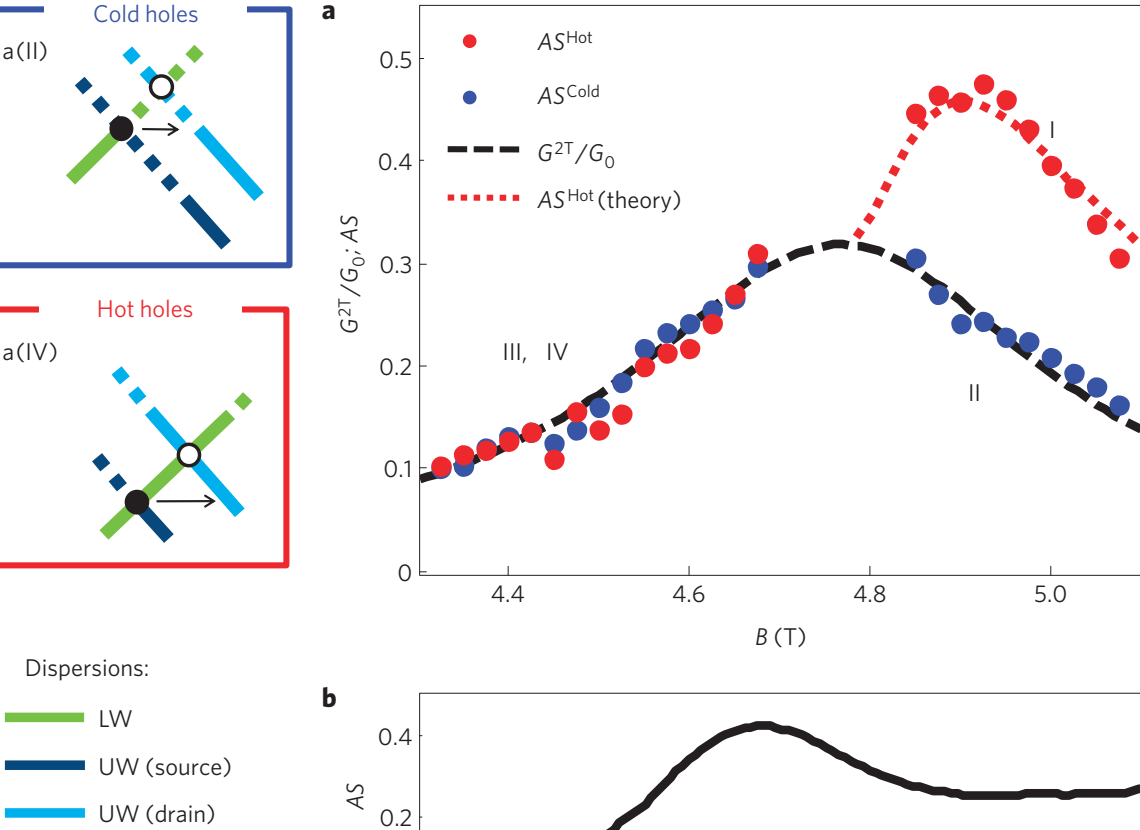

b

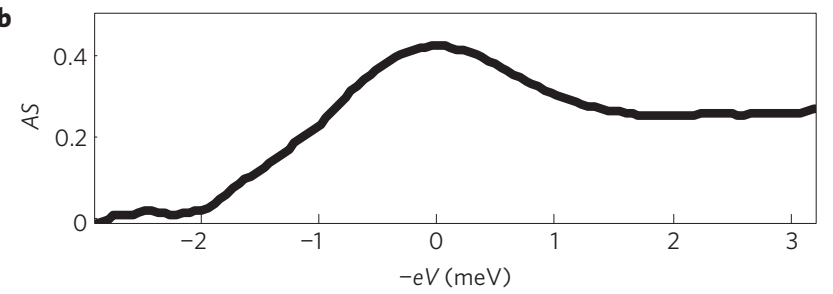

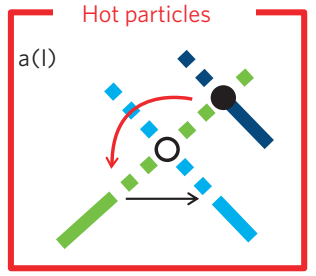
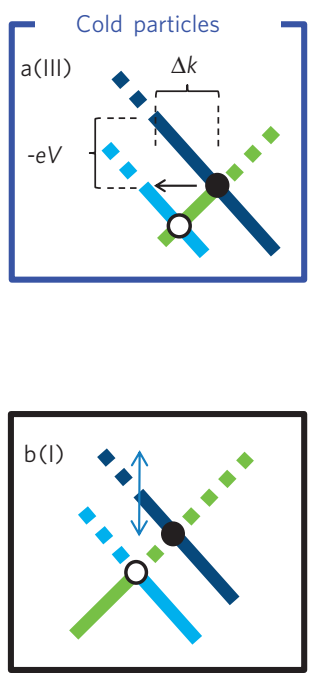

Figure 3 | Asymmetry of cold and hot particles. a, The cold asymmetry shows a single peak structure centred at $B^{L}$, with a diminishing asymmetry resulting from drain detuning. The asymmetry of cold particles is equal to the (normalized) two-terminal conductance. The hot hole $\left(B<B^{\mathrm{L}} \approx 4.77 \mathrm{~T}\right)$ asymmetry is identical to the cold particle case. Hot particle asymmetry shows a high peak, indicating energetic particles do relax. Assuming thermalization of the distribution function after the relaxation, we obtain an excellent agreement with the measurement. Insets: Intersections of upper-wire and lower-wire dispersions near $B=B^{\mathrm{L}}$. At the source, both a momentum shift $\Delta k$ and an energy shift $-e V$ are induced, and tunnelling between states with equal energy and momentum is allowed (dark circles). At the drains only the shift in momentum remains, giving rise to drain detuning. The dispersions overlap at states that are either occupied or unoccupied on both wires (white circles), disallowing momentum and energy conserving tunnelling. Black arrows indicate the momentum mismatch for particles tunnelling at the drains. Hot particles relax (red arrow in a(I)) before being collected by the drains. $\mathbf{b}$, The energy dependence of the asymmetry when $B=B^{L}$. Inset: the measurement leading to $\mathbf{b}$, when the upper wire at the source is shifted in energy by the application of $V$, while the magnetic field is kept at $B=B^{L}$, leading to no detuning at the drains.

Here $f_{0}(\varepsilon)$ is the equilibrium distribution function and $f_{i}(\varepsilon)$ denotes the distribution functions in the lower wire for left-movers $(i=1$ to the right and $i=2$ to the left of the source) and right-movers $(i=3)$, see Fig. $1 b$; for definiteness, we consider injection of leftmoving particles from the source. The detailed theory ${ }^{9,20}$ of electron energy exchange implies that equilibration occurs predominantly between quasiparticles moving in the same direction. Assuming that the energy equilibration length between electrons moving in the same direction is shorter than the distance between the source and drain terminals, we identify $f_{i}(\varepsilon)$ with Fermi distribution functions having constant chemical potentials $\mu_{i}$ and temperatures $T_{i}$ throughout the respective domain of the lower wire. We find these six parameters from balance equations for the charge and energy currents at the source and (two) drain electrodes (yielding six equations in total). For example, the corresponding balance equations at the source read

$$
I_{2}-I_{1}=I_{\mathrm{s}} \quad \text { and } \quad P_{2}-P_{1}=P_{\mathrm{s}}
$$

with

$$
\begin{array}{r}
I_{\mathrm{S}}=\left(G_{0} / e\right) \int T(\Delta k(\varepsilon))\left[f_{\mathrm{U}}(\varepsilon)-f_{1}(\varepsilon)\right] \mathrm{d} \varepsilon \\
P_{\mathrm{S}}=\left(G_{0} / \mathrm{e}^{2}\right) \int T(\Delta k(\varepsilon))\left[f_{\mathrm{U}}(\varepsilon)-f_{1}(\varepsilon)\right] \varepsilon \mathrm{d} \varepsilon
\end{array}
$$

Here, the subscript $U$ stands for the upper wire. The junction transmission probability $T$ in equation (5) depends on energy $\varepsilon$ through the momentum detuning between the dispersions of the two wires $\Delta k(\varepsilon)$. We find the transmission probabilities at the source and drains based on two-terminal zero-bias measurements, in which the momentum detuning between the two wires is directly controlled by the applied magnetic field $B$ (see Supplementary Information for details).

At small bias, the three pairs of balance equations (of which the first pair is given by equations (3)-(5)) can be solved analytically. Finding the drain currents in this regime, we confirm the validity of equation (2). At higher biases we resort to a numerical solution of the balance equations. We find relatively small shifts of the chemical potentials $\mu_{i}$, of order $1 \%$ of the applied bias, but a significant increase in the effective temperatures reaching approximately $1 \mathrm{~K}$ per applied $1 \mathrm{mV}$ source bias. On the basis of the resulting distribution functions, we evaluate the differential conductances entering equation (2). Despite the simplicity of our model, we obtain quantitative agreement of the evaluated asymmetry with the experimental data, see Fig. 3a. Combined with the qualitative difference between the measured $A S^{\text {hot }}$ for hot-particle and hothole injection, this further corroborates the conclusion about the particle-hole asymmetry: energetic particles relax through the excitation of $\mathrm{p}-\mathrm{h}$ pairs, rapidly reaching a fully thermalized distribution function, whereas energetic holes travel along the quantum wire without appreciable relaxation.

As stated, the above theory is applicable under the assumption of weak interactions, whereas in practice electrons in the measured system are notably interacting, with a Luttinger parameter $g \approx 0.55$. Nonetheless, we obtain excellent agreement between the theoretical 
prediction and the measurements, suggesting the asymmetry of energetic particles is weakly dependent on interactions. In the Supplementary Information we suggest a set of equations generalizing the above description to include strong interactions, which indeed reproduce such a weak dependence on the strength of interactions. However, we would like to stress that this generalization is not derived from first principles. Indeed, our work emphasizes a significant gap in the theoretical understanding of interacting 1D systems in that a complete microscopic description of kinetics and energy relaxation in the strongly interacting limit does not exist at present.

\section{Methods}

The double-wire system is fabricated by the cleaved-edge overgrowth method ${ }^{21}$ (see Fig. 1b). The electrons in the upper wire are confined to a 20-25-nm-wide well in the growth direction and a triangular potential approximately $20 \mathrm{~nm}$ wide perpendicular to the cleave direction. The electrons in the lower wire are confined to a $30 \mathrm{~nm}$ well in the growth direction and a similar triangular potential perpendicular to the cleave plane. The barrier separating the wires is $6 \mathrm{~nm}$ wide and $300 \mathrm{mV}$ high. Two top gates are used to electrostatically deplete the upper wire beneath them while leaving the lower wire continuous, thus defining three junctions. The middle junction, termed the 'source', can have a length of $6-40 \mu \mathrm{m}$, determined by the distance between the two gates. The junctions on the right and left sides of the source, termed the right and left drains, are of millimetre length. We measure the differential conductance $\partial I / \partial V_{S D}$ between the source and each of the drains using standard lock-in techniques. Typically $\mathrm{d} V_{\mathrm{SD}}$ is $14 \mu \mathrm{V}$ and the modulation frequency is a few hertz. The measurements are taken in a ${ }^{3} \mathrm{He}$ refrigerator at $T=0.25 \mathrm{~K}$. In practice, a bias $V_{S-D}$ is imposed between the source and drains junctions. However, almost the entire potential drop takes place at the short source junction, because it constitutes the dominant resistance in the circuit. Therefore, $V \approx V_{\text {S-D }}$ at the source region and $V \approx 0$ at the drains.

Received 26 January 2010; accepted 19 April 2010; published online 30 May 2010

\section{References}

1. Auslaender, O. M. et al. Spin-charge separation and localization in one dimension. Science 308, 88-92 (2005).

2. Steinberg, H. et al. Charge fractionalization in quantum wires. Nature Phys. 4, 116-119 (2008).

3. Deshpande, V. V. \& Bockrath, M. The one-dimensional Wigner crystal in carbon nanotubes. Nature Phys. 4, 314-318 (2008).

4. Deshpande, V. V., Bockrath, M., Glazman, L. I. \& Yacoby, A. Electron liquids and solids in one dimension. Nature 464, 209-216 (2010).

5. Giamarchi, T. Quantum Physics in One Dimension (International Series of Monographs on Physics, Oxford Science Publications, Clarendon Press, 2004).

6. Pines, D. \& Nozières, P. The Theory of Quantum Liquids 3rd edn (Perseus, 1999).
7. Anderson, P. W. The 'strange metal' is a projected Fermi liquid with edge singularities. Nature Phys. 2, 626-630 (2006).

8. Nozières, P. The effect of recoil on edge singularities. J. Phys. I France 4, 1275-1280 (1994)

9. Khodas, M., Pustilnik, M., Kamenev, A. \& Glazman, L. I. Fermi-Luttinger liquid: Spectral function of interacting one-dimensional fermions. Phys. Rev. B 76, 155402 (2007).

10. Auslaender, O. M. et al. Tunneling spectroscopy of the elementary excitations in a one-dimensional wire. Science 295, 825-828 (2002).

11. Pham, K-V., Gabay, M. \& Lederer, P. Fractional excitations in the Luttinger liquid. Phys. Rev. B 61, 16397 (2000).

12. Heeger, A. J., Kivelson, S. A., Schreiffer, J. R. \& Su, W-P. Solitons in conducting polymers. Rev. Mod. Phys. 60, 781-850 (1988).

13. Kivelson, S. A. Electron fractionalization. Synth. Met. 125, 99-106 (2001).

14. Fisher, M. P. A. \& Glazman, L. I. in Transport in One-Dimensional Luttinger Liquid, in Mesoscopic Electron Transport (eds Sohn, L. L., Kouwenhoven, L. P. \& Schoen, G.) (NATO ASI Series, Vol. 345, Kluwer Academic, 1997).

15. Safi, I. \& Schulz, H. J. Transport in an inhomogeneous interacting one-dimensional system. Phys. Rev. B 52, R17040 (1995).

16. Le Hur, K., Halperin, B. I. \& Yacoby, A. Charge fractionalization in nonchiral Luttinger systems. Ann. Phys. 323, 3037-3058 (2008).

17. Orgad, D. et al. Evidence of electron fractionalization from photoemission spectra in the high temperature superconductors. Phys. Rev. Lett. 86, 4362-4365 (2001)

18. Berg, E., Oreg, Y., Kim, E-A. \& von Oppen, F. Fractional charges on an integer quantum Hall edge. Phys. Rev. Lett. 102, 236402 (2009).

19. Imambekov, A. \& Glazman, L. I. Universal theory of nonlinear Luttinger liquids. Science 323, 228-231 (2009).

20. Pereira, R. G., White, S. R. \& Affleck, I. Spectral function of spinless fermions on a one-dimensional lattice. Phys. Rev. B 79, 165113 (2009).

21. Yacoby, A., Stormer, H. L., Baldwin, K. W., Pfeiffer, L. N. \& West, K. W. Magneto-transport spectroscopy on a quantum wire. Solid State Commun. 101, 77-81 (1997).

\section{Acknowledgements}

We would like to thank B. I. Halperin and K. Le Hur for helpful discussions. This work is supported by the NSF under contract DMR-0707484 at Harvard University, by the DOE under contract DE-FG02-08ER46482 at Yale University and by DIP at Freie Universität Berlin.

\section{Author contributions}

G.B., H.S. and A.Y. planned and carried out the experiments. L.N.P. and K.W.W. fabricated the samples. L.G., F.v.O., A.Y. and G.B. worked out the theory.

\section{Additional information}

The authors declare no competing financial interests. Supplementary information accompanies this paper on www.nature.com/naturephysics. Reprints and permissions information is available online at http://npg.nature.com/reprintsandpermissions. Correspondence and requests for materials should be addressed to A.Y. 